Reprod. Nutr. Dévelop., 1986, 26 (2 A), 393-407.

\title{
Tolérance physiologique de l'œuf de carpe (Cyprinus carpio L.) à un choc acide appliqué au cours de l'activation, ou pendant les premières divisions de segmentation
}

\author{
T. DESIRA ( $\left.{ }^{(}\right)\left({ }^{2}\right)$, P. ROUBAUD $\left({ }^{*}\right)\left({ }^{1}\right)\left({ }^{2}\right)$, Catherine CHAILLOU (1) ( $\left.{ }^{2}\right)$, \\ Ch. GILLET ( $\left.{ }^{3}\right)$, Pierrette REINAUD ( ${ }^{1}$ )
}

avec la collaboration technique : Installations piscicoles expérimentales :

G. FAYMENDI, P. LE BRENN, E. LETELLIER, D. MARIE. Entretien des Laboratoires : Marie-Louise PETINON et Rose-Marie PLACID. Réalisation des figures et dactylographie : M. WEBER et S. LAURENT.

(1) Station de Physiologie animale, Groupe "Embryologie des Poissons ", I.N.R.A. Jouy-en-Josas 78350 France.

(2) Equipe de Biologie du Développement, Université Paris VII, 2 place Jussieu 75251 Paris Cedex 05.

(3) Station d'Hydrobiologie lacustre,

I.N.R.A. 75 avenue de Corzent, 74203 Thonon-les-Bains, France.

Summary. Physiological tolerance of carp (Cyprinus carpio L.) eggs in response to acid shock during activation or early cleavage.

The tolerance of common carp eggs to a short acid shock (10-30 min) was studied by plunging the eggs in a strongly buffered acid solution during their activation or cleavage.

Egg tolerance increased during activation; its cyclic pattern then followed the rhythm of segmentation division. Egg sensitivity depended on shock duration, $\mathrm{pH}$ value and buffering strength of the acid solution. Egg tolerance was very high at the end of epiboly. During the first cycle of tolerance variation, an increase in shock strength (low pH) or duration caused asymmetrical widening of the cyclic sensitivity peaks.

The tolerance cycle did not appear to fluctuate in all of the trials.

The results have been interpreted in relation to some processes involved in egg activation (water inflow through the shell, variation in membrane permeability, calcium wave within the cytoplasm) or to the existence of an acid-sensitive phase in the blastomere cell cycle during synchronous cleavage.

\section{Introduction.}

La sensibilité des poissons d'eau douce à l'acidification des eaux - l'une des principales menaces qui pèsent sur l'environnement (Fromm, 1980 ; SNSF Pro-

$\left(^{*}\right)$ à qui toute correspondance doit être adressée : Equipe de Biologie du Développement, Anatomie comparée, Université Paris VII, 2 place Jussieu 75251 Paris Cedex 05. 
ject, 1980 ; Haines, 1981 ; Howells et al., 1983) - tient pour l'essentiel à la sensibilité du développement embryonnaire et larvaire. Tous les stades de ce développement ne sont pas également sensibles : ainsi, l'embryon œillé est plus tolérant que l'embryon éclos, sans doute en raison du rôle protecteur de la coque et de l'espace périvitellin (Peterson et al., 1980 ; Johansson et al., 1981 ; Daye, 1980 ; Rombough, 1983 ; Geen et al., 1985). Si un tel rôle existe, la fécondation doit aussi constituer une période sensible, car l'action des eaux acides est alors favorisée par l'existence de l'ouverture micropylaire (Kudo, 1980) et surtout par l'appel d'eau qui est lié au gonflement de l'œuf (Devillers et al., 1953 ; Thomopoulos, 1953 ; Soin, 1977, Peterson et Martin-Robichaud, 1982).

Cependant, les liquides spermatique et ovarien excercent, de par leur $\mathrm{pH}$ alcalin (Plouidy, 1982), un rôle protecteur, surtout efficace, pour des espèces comme la Carpe, qui pondent dans des eaux immobiles et peu profondes (Soin, 1977). Dans les conditions naturelles, c'est la course de vitesse qui s'engage entre la dispersion des liquides gamétiques et la mise en place du système coqueespace périvitellin qui détermine ce que nous avons appelé la tolérance écologique de l'espèce. Au contraire, la tolérance physiologique propre des gamètes et de la fécondation ne peut être étudiée que si l'on supprime les effets protecteurs des liquides gamétiques, ce qui ne peut être obtenu qu'en utilisant des milieux fortement tamponnés (Roubaud et al., 1984).

Dans ce qui suit, nous nous proposons de comparer, sur l'œuf de la carpe commune, la sensibilité physiologique de la fécondation à celle des premiers cycles cellulaires de la segmentation.

Matériel et méthodes. (voir tabl. 1) :

Aux différents âges embryonnaires étudiés, les œufs subissent un choc acide dont les effets sont évalués en mesurant les taux de survie aux stades de la fin de l'épibolie, de l'embryon œillé, ou de pré-éclosion $\left(1{ }^{\mathrm{e}}, 2^{\mathrm{e}}\right.$ et $3^{\mathrm{e}}$ jour $\mathrm{d}^{\prime}$ incubation à $\left.20^{\circ} \mathrm{C}\right)$.

Le critère de discrimination des œufs morts est la coagulation du vitellus.

Le choc acide est caractérisé par les paramètres suivants : - l'âge de l'embryon au début du choc (a) est compté à partir du moment $(a=0)$ de la dispersion des gamètes dans le dilueur de fécondation; - la durée du choc et son $\mathrm{pH} ;-$ la concentration de la solution acide testée.

Les agents tampons de $\mathrm{pH}$ sont utilisés au voisinage de leur $\mathrm{pK}$ d'ionisation (Von Rauen, 1964). Suivant les $\mathrm{pH}$ choisis, nous avons utilisé : I'acide acétique $(\mathrm{pH} 3,5$ à 5,0); la 4-3 amino-propylmorpholine $(\mathrm{pH} \mathrm{6,2)}$; l'éthylène diamine $(\mathrm{pH}$ $6,6-7,0)$.

L'entrée et la sortie de choc sont obtenues en plongeant d'un coup les œufs dans le dilueur de choc, puis en les transportant en eau courante après lavage éventuel dans une solution de Tris (tris-hydroxyméthyl-aminométhane) à $25 \mathrm{mM}$ ajustée par $\mathrm{HCl}$ au $\mathrm{pH} \mathrm{8,2.}$

La production des gamètes, la fécondation et l'incubation sont réalisées suivant Roubaud et al. (1984). 


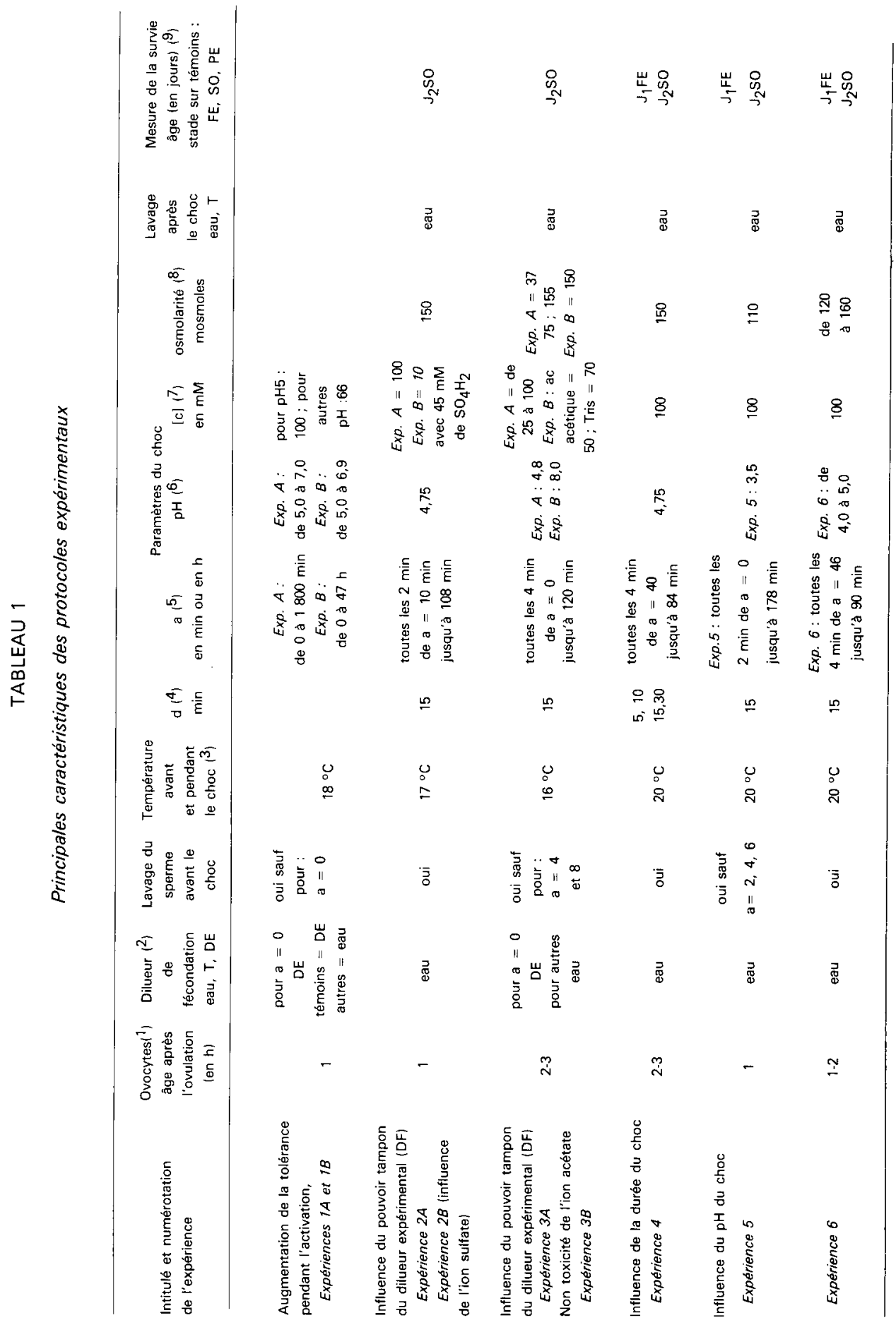




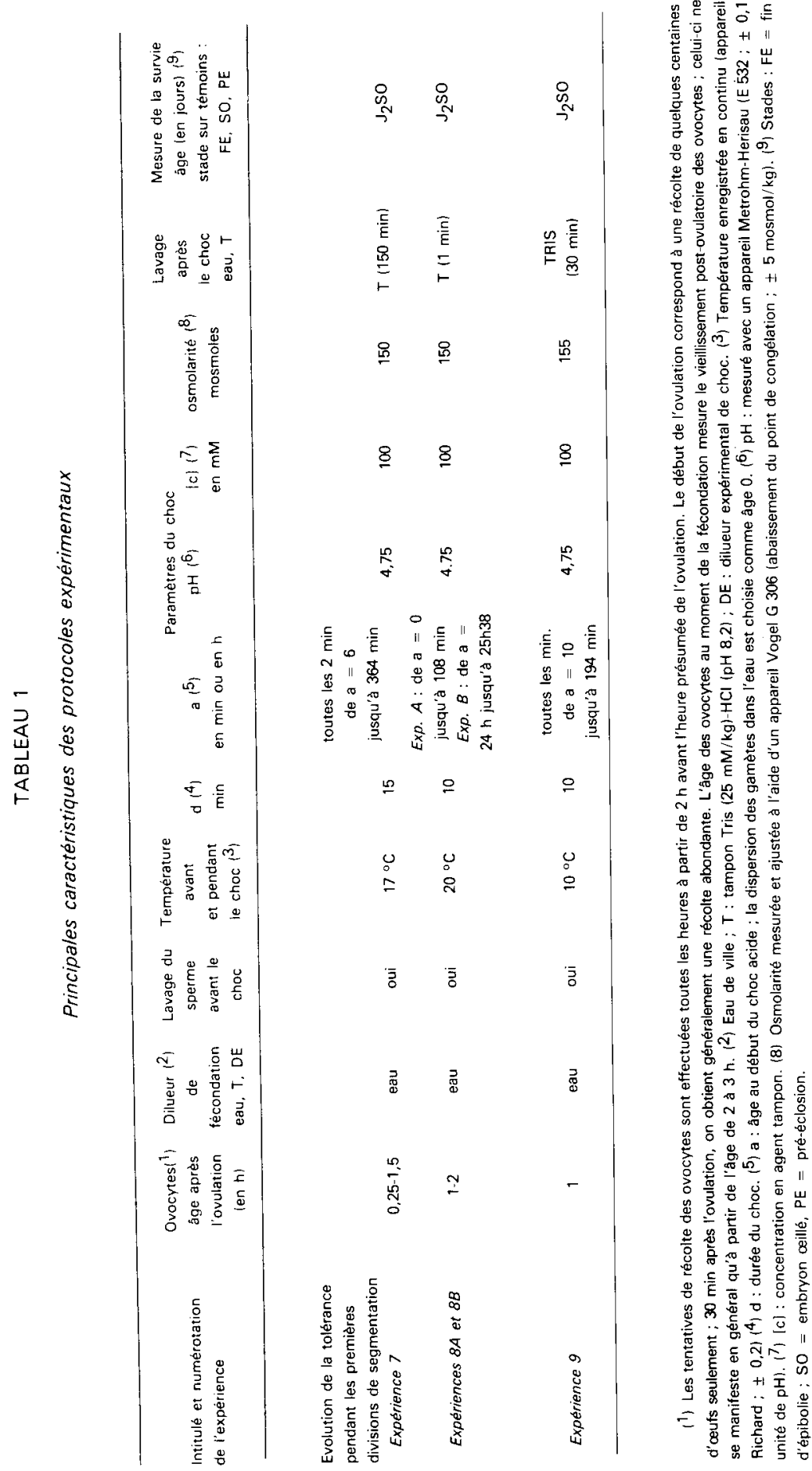


La fécondation est réalisée soit en eau de ville (eau), soit dans un tampon Tris $(25 \mathrm{mM})-\mathrm{HCl}$ de $\mathrm{pH} 8,2$, soit dans un dilueur expérimental de choc acide.

Les températures de fécondation et d'incubation sont enregistrées en continu (appareil Richard, précision $\pm 0,2^{\circ} \mathrm{C}$ ).

Le $\mathrm{pH}$ des solutions est mesuré avec un appareil Metrohn-Herisau, E 532, précision $\pm 0,1$ unité de $\mathrm{pH}$.

L'osmolarité mesurée est ajustée à l'aide d'un appareil Vogel G 306, précision \pm 5 mosM.

Les stades de développement au moment de la mesure du taux de survie sont la fin d'épibolie, le stade de l'embryon œillé et le stade de prééclosion.

Neuf séries d'essais ont été réalisées suivant les protocoles décrits dans le tableau 1. Elles utilisaient chacune (et dans l'expérience $n^{\circ} 1$, les essais $1 \mathrm{~A}$ et 1B) des ovocytes produits par des femelles différentes.

\section{Résultats.}

Les minutes qui suivent la fécondation sont celles d'une grande sensibilité au choc acide. La durée de la période de sensibilité initiale dépend du $\mathrm{pH}$ (fig. 1) et du pouvoir tampon (fig. 3, 4 et 5). (Dans l'expérience 2B, fig. 4, I'acide sulfurique n'exerce aucun pouvoir tampon).

Pendant l'activation la tolérance augmente rapidement (fig. 1, 2, 3, 5, 11, 13, 15). Dans plusieurs essais, la tolérance ainsi acquise parait se conserver dans la suite du développement (fig. 1,3,15), dans d'autres, elles ne se maintient pas totalement (fig. 2-5).

Avec un $\mathrm{pH}$ très acide $(\mathrm{pH} 3,5)$, un pic de tolérance partielle apparaît au 1er jour d'incubation (fig. 6), mais tous les œufs sont morts un jour plus tard.

Dans plusieurs essais, un caractère cyclique de l'évolution de la tolérance apparaît : sur un premier cycle dans les essais $n^{\circ} 4$ et 5 (fig. 7, 8, 9, 10), et sur 6 cycles successifs dans l'expérience $n^{\circ} 7$ (fig. 11, 12). Dans cette expérience, la durée moyenne d'un cycle est 44 min (fig. 12) ; après un premier maximum, la tolérance baisse progressivement. Les rythmes de la variation cyclique de tolérance dépendent des températures d'incubation.

D'après nos observations, le vitellus des œufs non fécondés met moins d'un jour à coaguler ; il est donc possible, pour des chocs appliqués à partir de l'âge $a=1$ jour, de ne tenir compte que des œufs comptés vivants avant le choc : à l'âge de 1 jour, l'embryon est devenu tolérant au choc acide (fig. 14).

Lorsque, au cours du premier cycle d'oscillation de la tolérance, le choc acide se prolonge (fig. 7, 8), ou lorsque l'acidité du dilueur de choc augmente (fig. 9, 10), les maxima de tolérance ne correspondent plus qu'à une tolérance partielle. D'autre part, lorsque le $\mathrm{pH}$ du dilueur passe de 4,5 à 4,25 et 4,0 (fig. 9 et 10), le premier pic de sensibilité cyclique s'élargit dissymétriquement vers les âges les plus élevés : en effet, alors que les courbes obtenues à ces différents $\mathrm{pH}$ baissent au même moment, elles tendent à se relever d'autant plus tard que le $\mathrm{pH}$ du choc est plus bas.

Un effet analogue apparaît aussi (fig. 7 et 8 ), lorsque la durée du choc toxique passe de 15 à 30 min (fig. $n^{\text {os }} 7$ et 8 ). De même, dans l'expérience $n^{\circ} 3$ (fig. 

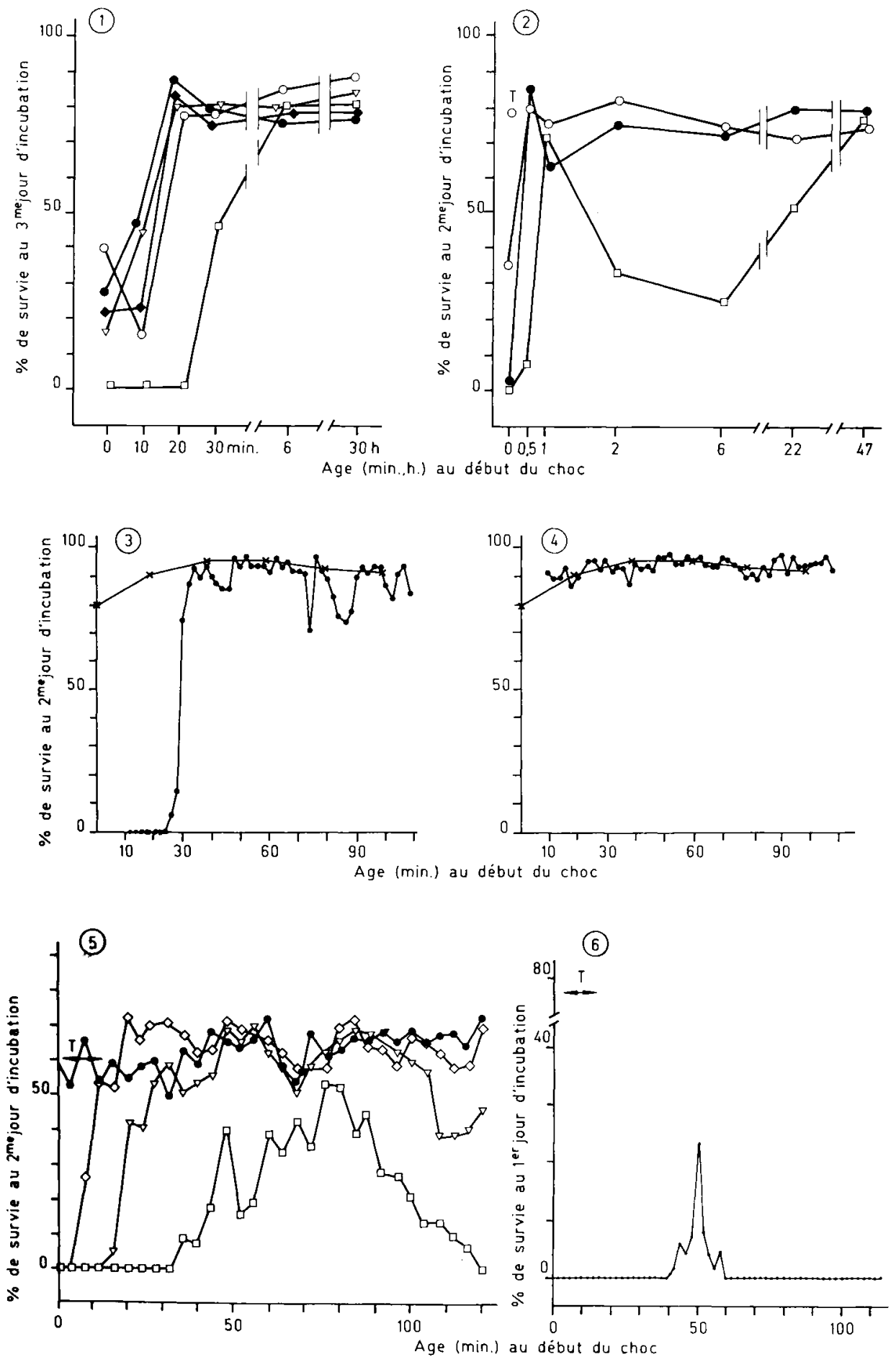
5), la durée de la phase sensible qui suit la fécondation est d'autant plus grande que la concentration en agent tampon est plus élevée.

Des effets différés des chocs sur la survie se manifestent entre le $2^{\mathrm{e}}$ et le $3^{\mathrm{e}}$ jour d'incubation.

$\mathrm{Au} \mathrm{pH} \mathrm{8,2,} \mathrm{sous} \mathrm{sa} \mathrm{forme} \mathrm{ionique,} \mathrm{l'acide} \mathrm{acétique} \mathrm{n'est} \mathrm{pas} \mathrm{toxique} \mathrm{à} \mathrm{la}$ concentration de $50 \mathrm{mM}$. Au pH 4,8 au contraire, un mélange à parties égales des formes ionique et moléculaire apparaît comme toxique pendant les périodes de sensibilité.

\section{Discussion.}

Critique des protocoles expérimentaux.

1) Pouvoir tampon et toxicité de l'acide acétique. - Les chocs acides auxquels nous avons eu recours pour l'étude de la tolérance physiologique aux bas $\mathrm{pH}$ sont en réalité des chocs complexes pour lesquels divers facteurs doivent être pris simultanément en considération : le $\mathrm{pH}$, l'osmolarité, la nature de l'espèce ionique utilisée comme agent tampon, le pouvoir tampon de la solution.

D'autre part, l'expérience 3B (fig. 5) démontre l'innocuité de l'ion acétate au $\mathrm{pH} 8$, mais les effets spécifiques de la molécule d'acide acétique sont difficiles à départager de ceux du $\mathrm{pH}$ lors d'un choc par une solution acide acétique-acétate

FIG. 1. - Exp. 1A (cf. tabl. 1) - Augmentation au cours de l'activation et en fonction du pH, de la tolérance physiologique de l'œu fe carpe à un choc acide de concentration en agent tampon $100 \mathrm{mM}$ au pH 5 et $66 \mathrm{mM}$ aux autres $\mathrm{pH}$ et de durée $30 \mathrm{~min}$.

$\mathrm{pH} 6,8 \longrightarrow \mathrm{pH} 6,6 ; \nabla-\square: \mathrm{pH} \mathrm{6,2; \square — \square}: \mathrm{pH} \mathrm{5,0}$.

FIG. 2. - Exp. 18 (cf. tabl. 1) - La tolérance acquise en fin d'activation, après un choc acide, ne se maintient pas nécessairement au cours de la segmentation. Durée du choc : 30 min ; concentration en agent tampon: $0,1 \mathrm{M}$; $\mathrm{T}$ : pourcentage de survie moyen sur les lots témoins;

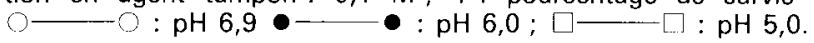

FIG. 3 et 4. - Exp. 2A, $2 B$ (cf. tabl. 1) - Influence du pouvoir tampon de la solution de choc sur l'évolution de la tolérance à un choc acide $(\mathrm{pH} \mathrm{4,75)}$ appliqué au cours de l'activation ou au début de la période de segmentation. $\mathrm{X}-\mathrm{X}$ : pourcentage de survie des lots témoins fécondés en même temps que les lots expérimentaux dont l'âge est indiqué en abscisse. Fig. 3 : Dilueur expérimental $\mathrm{NaOH}$-acide acétique $100 \mathrm{mM}$; Fig. 4 : Dilueur expérimental $\mathrm{NaOH}$-acide acétique $10 \mathrm{mM}$. acide sulfurique $45 \mathrm{mM}$.

FIG. 5. - Exp. 3A, 3B (ct. tabl. 1). - Innocuité de l'ion acétate et influence du pouvoir tampon de la solution de choc sur l'évolution de la tolérance à un choc acide appliqué au cours de l'activation et au début de la segmentation. - pH 4,80: tampon acide acétique - $\mathrm{NaOH}$, concentration en acide acétique $(\mathrm{mM}): \square-\square 100 ; \nabla-\square 50 ; \diamond-\square 25 .-\mathrm{pH} 8:$ tampon acide acétique-Tris, concentration en acide acétique : $50 \mathrm{mM}$.

FIG. 6. - Exp. 5 (cf. tabl. 1). - Tolérance physiologique à un choc acide de $\mathrm{pH} 3,5$ et de concentration en agent tampon $0,1 \mathrm{M}$. Choc appliqué pendant $15 \mathrm{~min}$ au cours de l'activation et au début de la segmentation. 

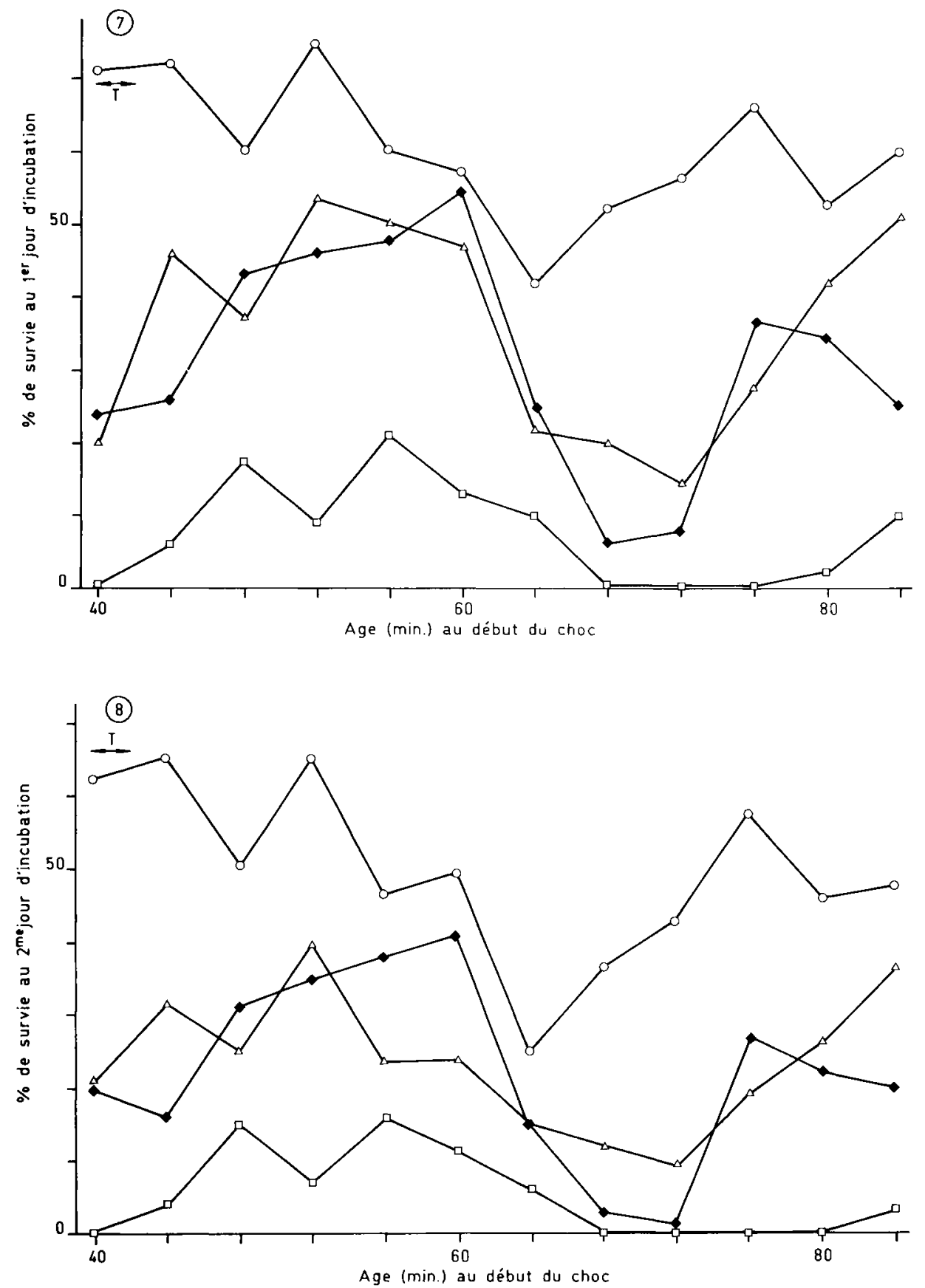

FIG. 7 et 8. - Exp. 4 (cf. tabl. 1). - Evolution, au début de la période de segmentation, de la tolérance en fonction de la durée du choc acide. $\mathrm{pH}$ du choc : 4,75, concentration en agent tampon

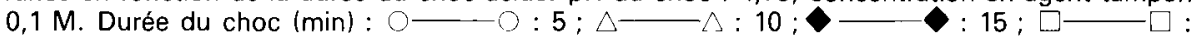
30. Fig. 7 et 8 : observation au 1 er et $2^{e}$ jour d'incubation. 

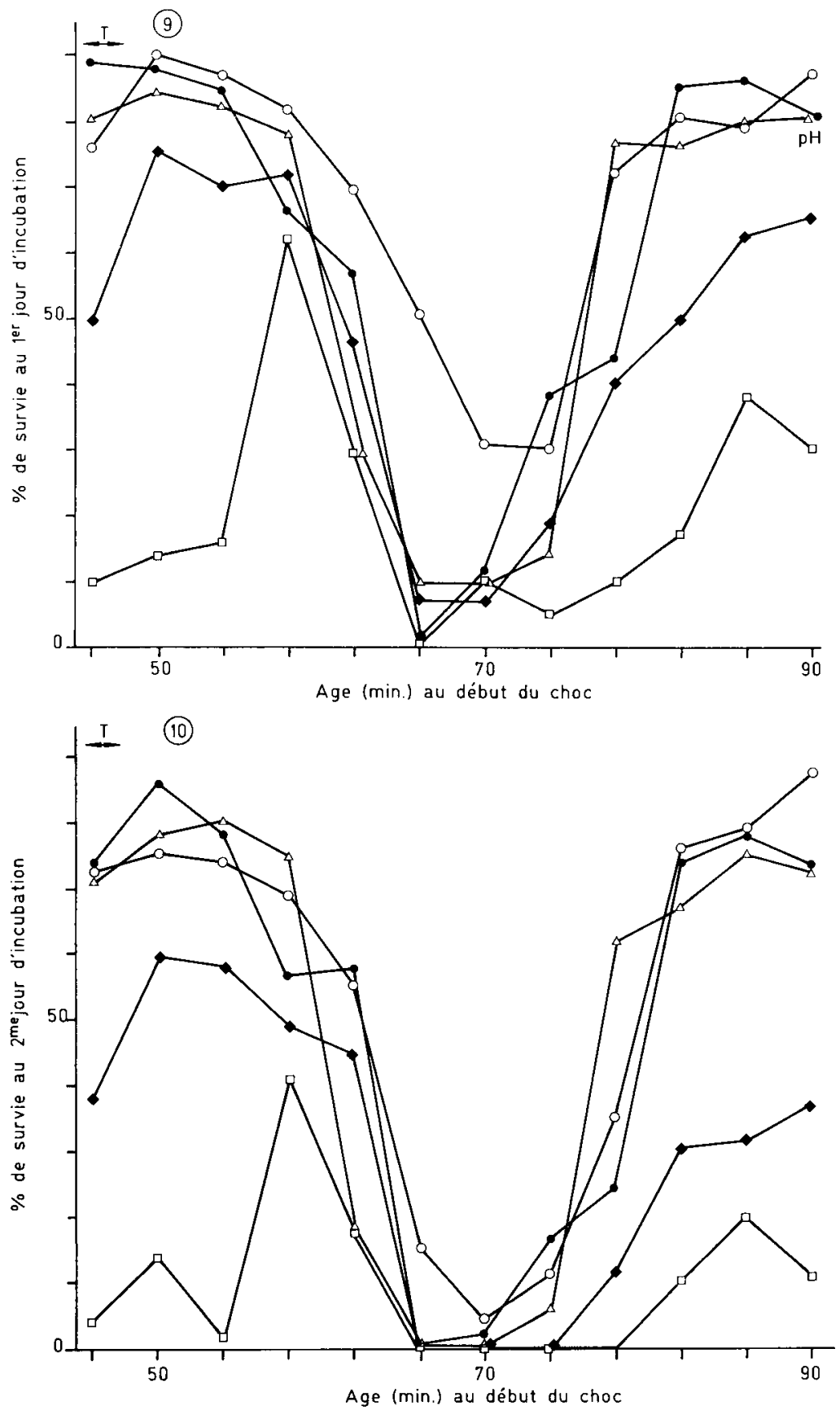

FIG. 9 et 10. - Exp. 6 (cf. tabl. 1). - Tolérance physiologique de l'ouf de carpe en fonction du $\mathrm{pH}$ d'un choc acide de concentration en agent tampon $0,1 \mathrm{M}$ et durée $15 \mathrm{~min}$. $\mathrm{pH}$ du choc: $\longrightarrow$ -

Fig. 9 et 10 : observation au $1^{\text {er }}$ et $2^{\mathrm{e}}$ jour $\mathrm{d}^{\prime}$ incubation. 

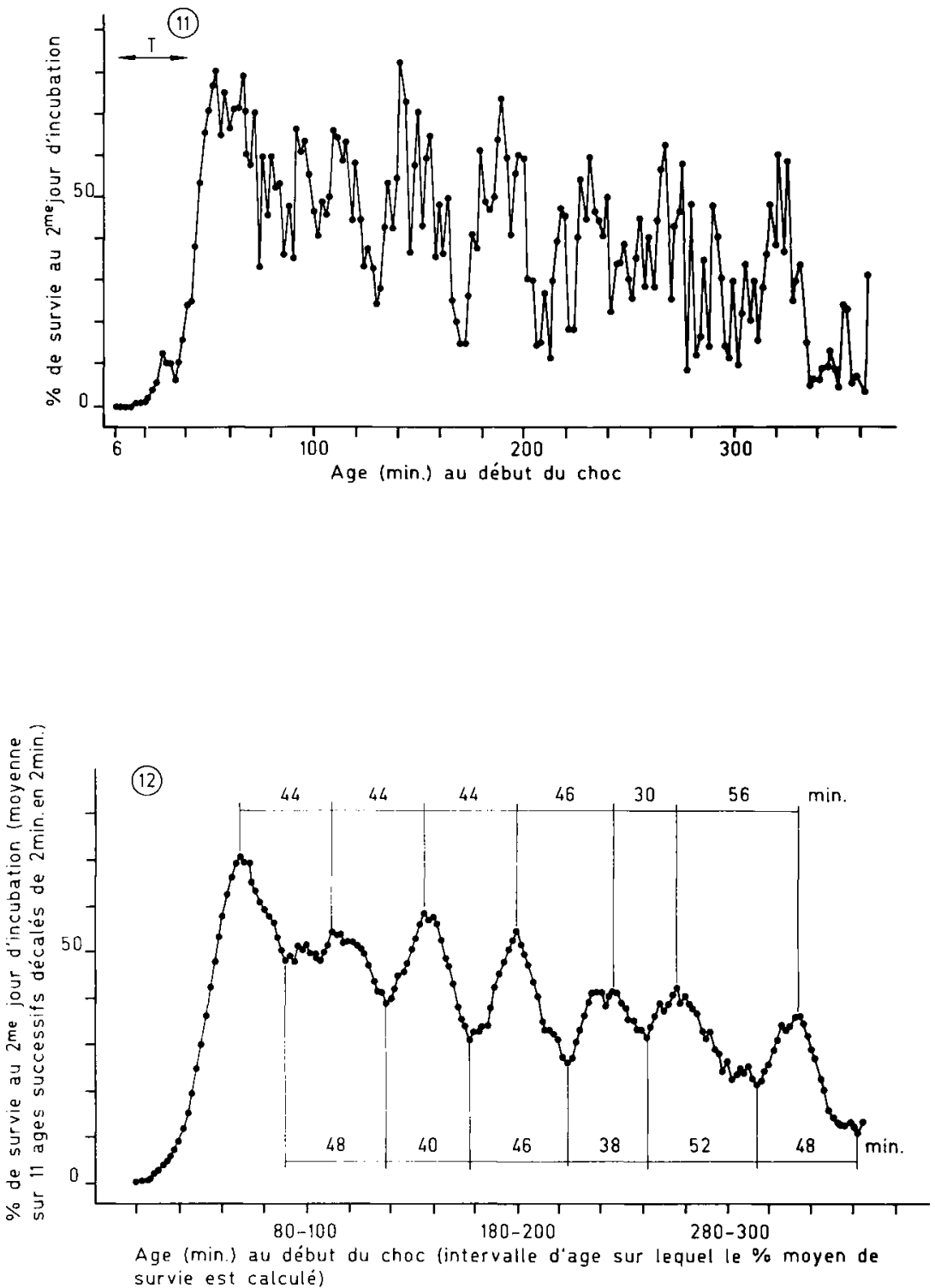

FIG. 11 et 12. - Exp. 7 (cf. tabl. 1). - Evolution, au cours de l'activation et des premières divisions de segmentation, de la tolérance physiologique de l'ceuf de carpe à un choc acide de $\mathrm{pH} 4,75$ et de concentration en agent tampon $0,1 \mathrm{M}$ et de durée $15 \mathrm{~min} \longrightarrow \longrightarrow$ \% de survie des lots témoins. Fig. $11: \%$ de survie au $2^{\mathrm{e}}$ jour $\mathrm{d}^{\prime}$ incubation ; Fig. $12: \%$ de survie moyen sur un intervalle d'âge de $20 \mathrm{~min}$, c c-a-d, sur 11 lots expérimentaux successifs d'âges, au début de choc, décalés de $2 \mathrm{~min}$ en $2 \mathrm{~min}$. 


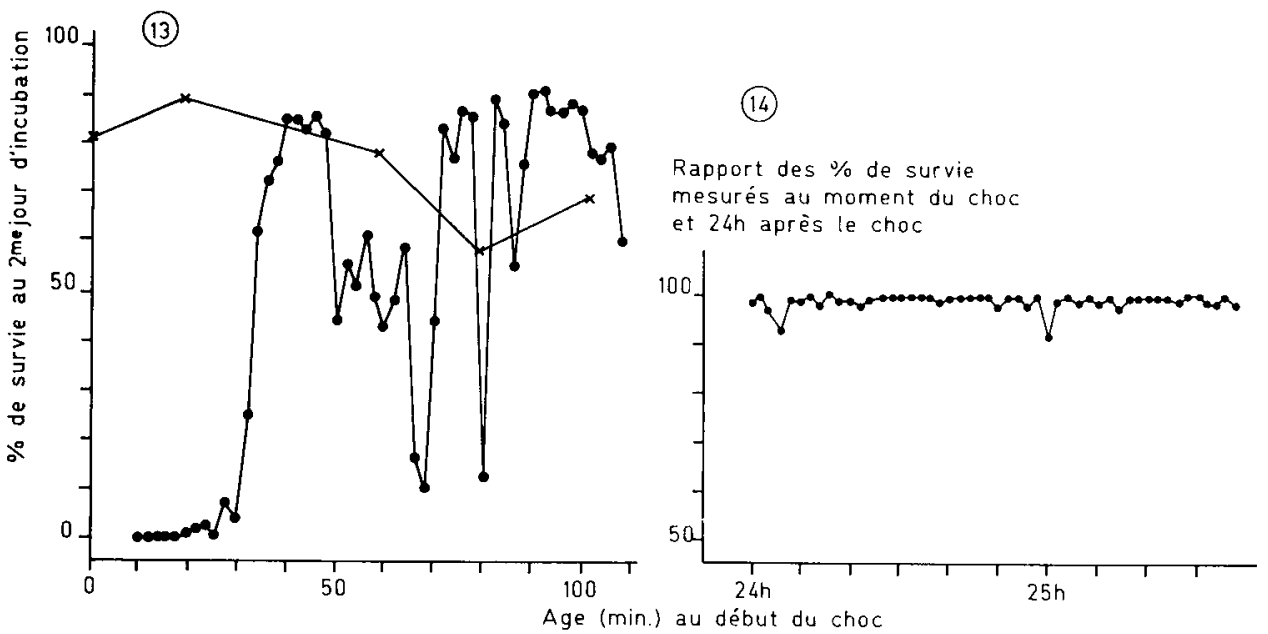

FIG. 13. - Exp. 8A (cf. tabl. 1). - Evolution, au cours de l'activation et du début de la segmentation, de la tolérance physiologique à un choc acide de $\mathrm{pH} 4,75$, de concentration en agent tampon $0,1 \mathrm{M}$ et de durée $10 \mathrm{~min}$. $\bigcirc \longrightarrow \mathrm{O}$ : lots expérimentaux ; $\mathrm{x}-\mathrm{x}$ : lots témoins fécondés en même temps que les lots expérimentaux dont les âges en début de choc sont indiqués en abscisse.

FIG. 14. - Exp. 8B. - Evolution, à la fin de l'épibolie, de la tolérance à un choc acide de $p H 4,75$, de concentration en agent tampon $0,1 \mathrm{M}$, et de durée $10 \mathrm{~min}$. Le $\%$ de survie, mesuré $24 \mathrm{~h}$ après le choc, est exprimé en valeur relative au \% de survie avant le choc.

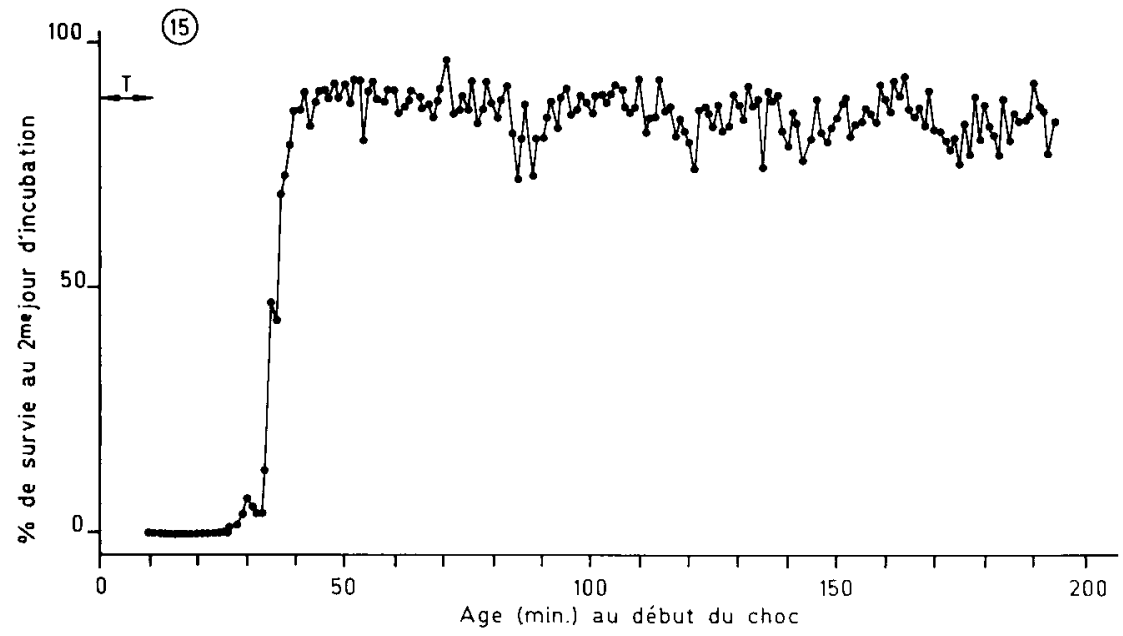

FIG. 15. - Exp. 9 (cf. tabl. 1). - Evolution, au cours de l'activation et du début de la segmentation, de la tolérance physiologique à un choc acide de $\mathrm{pH} 4,75$, de concentration en agent tampon $0,1 \mathrm{M}$ et de durée $10 \mathrm{~min}$. 
de $\mathrm{pH}$ 4,75. De même, l'expérience 2 (fig. 3 et 4) permet de comparer au pH 4,75, les effets d'une solution acétique $0,1 \mathrm{M}$ à ceux d'une solution de même $\mathrm{pH}$, et osmolarité, mais dont les $9 / 10^{e}$ de l'acide acétique ont été remplacés par de l'acide sulfurique; les effets du choc sont alórs supprimés. Cependant, il est difficile de départager ce qui est alors dû à la modification de la concentration en acide acétique moléculaire et ce qui est dû au changement de pouvoir tampon de la solution : sur Medaka adulte (Oryzias latipes), Josuka et Adachi (1979), ont

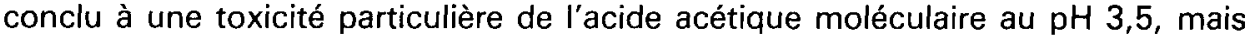
le rôle du pouvoir tampon, au moment de la fécondation, pourrait être déterminant sur l'œuf de Carpe (Roubaud et al., 1984).

2) Interférence du choc osmotique. - Dans nos expériences, les œufs ont été soumis, à l'entrée et à la sortie du choc, à des chocs osmotiques d'amplitude maximale 150 mosM. Les expériences 2B et 3B (fig. 4 et 5), montrent l'innocuité de ces chocs. Cependant, cette conclusion n'exclut pas que l'osmolarité de la solution de choc puisse interférer avec les effets de $\mathrm{pH}$, par exemple, en modifiant la perméabilité de la membrane vitelline (Potts et Rudy, 1969), ou le gonflement de l'œuf (Soin, 1977).

Sensibilité initiale au choc acide.

1) Evolution initiale de la sensibilité. - Dans les minutes qui suivent la fécondation, une tolérance non nulle apparaît d'autant plus tôt que l'intensité du choc diminue : pH plus élevé (fig. 1 et 2), pouvoir tampon plus faible (fig. $3,4,5$ ). $C^{\prime}$ est donc une période débutant au moment de la dispersion des gamètes et durant au maximum la durée d'un choc (15 min ou plus) qui représente le moment de plus grande sensibilité.

2) Interprétations physiologiques. - La pénétration du spermatozoïde n'est sans doute pas le processus sensibilisateur déterminant aux eaux acides. En effet, elle ne dure que 1 à 2 min dans le liquide ovarien (Kudo, 1980), et, moins de 8 min dans une solution à 150 mosM (Sjafei, non publié). Elle est donc terminée à des âges où, pourtant, la sensibilité aux pH acides est encore très grande (fig. 5 , $6,11,13,15)$.

L'appel d'eau lié au gonflement de l'œuf est susceptible de favoriser la pénétration des molécules acidifiantes et d'expliquer l'existence de la période de sensibilité initiale. D'après nos observations, dans l'eau à $20^{\circ} \mathrm{C}$ le gonflement de l'œuf de carpe est terminé, pour l'essentiel, après $10 \mathrm{~min}$. En solution saline, cependant, le gonflement de l'œuf de truite (Devillers et al., 1953), ou de perche (Thomopoulos, 1953) est retardé ; celui de l'œuf de carpe est non seulement retardé (Chaillou et al., non publié), mais il est aussi augmenté au total (Soin, 1977). La durée de la période de sensibilité initiale observée dans nos expériences pourrait donc correspondre à celle du gonflement de l'œuf. Cependant, le gonflement de l'œuf de Salmo salar (Peterson et Martin-Robichaud, 1982) ou de perche (Rask, 1983 ) est diminué en solution acide. L'hypothèse d'un rôle du gonflement dans la 
sensibilisation initiale aux eaux acides devra donc être testée en modifiant la composition saline avant et après le choc.

La perméabilité de la membrane vitelline augmente, chez Salmo salar, pendant quelques minutes après la fécondation pour décroître ensuite jusqu'à une valeur très basse (Potts et Rudy, 1969 ; Loefler, 1971). Cette évolution pourrait être déterminante dans celle de la sensibilité aux acides. La possibilité de modifier la perméabilité par une solution saline ou par du glucose (Potts et Rudy, 1969) devrait servir à tester cette hypothèse.

C'est la libération d'une onde calcique intra-cytoplasmique autopropagée qui est responsable du déclenchement de l'ensemble des processus de l'activation dans l'œuf de Médaka (Ridgway et al., 1977 ; Gilkey et al., 1978, 1981). En milieu acide (Gilkey, 1983), le seuil de déclenchement et d'autopropagation de cette onde serait augmenté, mais, une fois terminé le passage de l'onde sur la totalité de la surface de l'œuf, le développement pourrait se poursuivre normalement. Ainsi pourrait s'expliquer la sensibilité initiale de l'cuf aux bas $\mathrm{pH}$.

L'acquisition d'une tolérance élevée en fin d'activation pourrait s'interpréter simplement par la fin des processus sensibilisateurs mentionnés plus haut. A ces facteurs, pourrait s'ajouter le rôle exercé par le liquide périvitellin comme tampon de $\mathrm{pH}$ (Peterson, et al., 1980 ; Johanson et al., 1981).

Les fluctuations cycliques de la tolérance embryonnaire au cours de la segmentation présentent un rythme comparable à celui observé par Ignatieva (1974) pour le cycle des blastomères. Les premières divisions de segmentation sont remarquablement synchrones (Dondua et al., 1976 ; Rott, 1979, 1980) : il paraît donc logique d'attribuer les fluctuations de la tolérance embryonnaire à des variations sousjacentes de la tolérance cellulaire. Une telle correspondance a déjà été décrite pour la tolérance à un choc froid (Roubaud et al., 1985) ou à un choc chaud (Chaillou et al., non publié).

L'embryon âgé de 1 jour, contrairement à l'œuf en segmentation, montre une tolérance élevée au choc acide (fig. 14). Cette acquisition de tolérance, déjà observée pour les chocs chauds et froids (Jaoul et Roubaud, 1982) pourrait s'expliquer par la désynchronisation et le ralentissement cellulaires qui interviennent au moment de la transition blastuléenne (Roubaud et al., 1985).

Dans deux expériences (fig. 3 et 15) où les taux de survie sur les lots témoins sont particulièrement élevés $(90,3$ et $88 \%)$, les fluctuations cycliques de la tolérance $n^{\prime}$ apparaissent pas. La qualité des gamètes pourrait donc être un facteur déterminant de la tolérance au choc acide. Cependant, dans une autre expérience (fig. 9 et 10), la variation cyclique de tolérance apparaît avec des taux de survie sur témoin de $92 \%$.

Dans l'expérience $n^{\circ} 9$ (fig. 15), l'absence de fluctuation cyclique pourrait être due au lavage par une solution alcaline tamponnée à la sortie du choc. Peterson et al. (1980) ont montré que le $\mathrm{pH}$ du liquide périvitellin met plusieurs heures à atteindre une valeur d'équilibre lorsque l'œuf de Salmo salar est placé dans une eau acide non tamponnée. A l'inverse, on peut attendre que dans l'eau du robi- 
net, le liquide périvitellin reste encore acide un certain temps après la fin du choc et en prolonge ainsi les effets. Le lavage par une solution alcaline reviendrait alors à réduire la durée du choc.

L'élargissement dissymétrique des pics de sensibilité lorsque l'intensité du choc augmente (fig. 7 à 10), rejoint une observation déjà faite après un choc froid (Roubaud et al., 1985) : tout semble se passer comme si le séjour en milieu acide se traduisait par un retour en arrière progressif d'un processus cellulaire sousjacent, le ramenant au niveau qui est le sien au moment de la période de sensibilité maximale. Sur la nature de ce processus, on ne peut formuler que des hypothèses en l'absence d'interprétation physiologique des mécanismes de l'action du choc acide.

La formulation d'hypothèses explicatives de la présence des phases de sensibilité cyclique doit tenir compte des hypothèses avancées plus haut pour expliquer l'existence de la phase de sensibilité initiale. Le rôle de l'appel d'eau associé au gonflement de l'œuf ne peut plus être invoqué, puisque celui-ci est pratiquement terminé, pour l'œuf de carpe, après 10 min dans l'eau à $20^{\circ} \mathrm{C}$ (Chaillou et al., travail en cours). Cependant, des variations cycliques de la pénétration des molécules au contact de l'embryon, sont encore une éventualité, car Komm et Reznichenko (1963), ont montré que l'embryon de poisson est animé dès la fécondation, par des mouvements périodiques qui brassent le liquide périvitellin et favorisent les échanges entre l'embryon et son milieu.

Les deux autres hypothèses invoquées pour l'interprétation de la phase de sensibilité initiale ne peuvent, non plus, être exclues a priori : rien n'interdit de penser, en effet, que la perméabilité et la surface de la membrane périvitelline ne subissent pas des variations régulières au cours du cycle cellulaire des blastomères en segmentation. De même, le cycle cellulaire pourrait comporter une phase d'activation par une onde calcique autopropagée analogue à celle qui déclenche l'activation au moment de la fécondation.

Recu en avril 1985.

Accepté en novembre 1985.

Remerciements. - Nous remercions R. Billard, Marie-Hélène Magri et D. Marie qui nous ont apporté leurs conseils et leur aide pour la bibliographie. Nous remercions aussi Claire Joly qui a assuré une lecture critique du manuscrit.

Ce travail a bénéficié du soutien du Secrétariat à l'Environnement (Contrat I.N.R.A. 8340-170) et du C.N.R.S. (R.C.P 08-06-56).

\section{Références}

DAYE P. G., 1980. Attempt to acclimate embryos and alevins of atlantic salmon Salmo salar L., and rainbow trout Salmu gairdneri, to low pH. Can. J. Fish. Aquat. Sci., 37, 1035-1038.

DEVILLERS Ch., THOMOPOULOS A., COLAS J., 1953. Differenciation bipolaire et formation de l'espace périvitellin dans I'œuf de Salmo irideus. Bull. Soc. Zool., Fr. 78, 462-470.

DONDUA A. K., ROTT N. N., GORODILOV Y. N., 1976. Desynchronisation of cell divisions in the early development of axolotl, loach and salmon. Ontogenez, 8, 11-20. 
FROMM P. O., 1980. A review of some physiological and toxicological responses of freshwater fish to acid stress. Env. Biol. Fish., 5, 79-93.

GEEN G. H., NEILSON J. D., BRADFORD M., 1985. Effects of $\mathrm{pH}$ on the early development and growth and otolithe microstructure of chinook salmon, Oncorhynchus tshawytscha. Can. J. Zool., 63, 22-27.

GILKEY J. C., 1981. Mechanisms of fertilization in fishes. Am. Zool., 21, 359-375.

GILKEY J. C., 1983. Roles of calcium and $\mathrm{pH}$ in activation of the eggs of the medaka fish, Oryzias latipes. J. Cell Biol., 97, 669-678.

GILKEY J. C., JAFFE L. F., RIDGWAY E. R., REYNOLDS G. T., 1978. A free calcium wave traverses the activing egg of medaka Oryzias latipes J. Cell Biol., 76, 448-466.

HAINES T. A., 1981. Acidic precipitation and its consequences for aquatic ecosystems; a rewiev. Trans. am. Fish. Soc., 110, 669-707.

HOWELLS G. D., BROWN D. J. A. , SADLER K. 1983. Effects of acidity, calcium and aluminium on fish survival and productivity; a review. J. Sci. Food. Agric., 34, 559-570.

IGNATIEVA G. M., 1974. Temperature dependance of cleavage rates in carp, pike and white fish. Ontogenez, 5, 27-32.

JAOUL A., ROUBAUD P. 1982. Resistance de l'œuf de carpe commune (Cyprinus carpio L.) à des chocs thermiques chauds ou froids $J$. can. Zool., 60, 3409-3419.

JOHANSSON N., RUNN P., SOHTELL M., 1981. Perivitelline $\mathrm{pH}$ of salmonid eggs in relation to ambient pH. Water Res. Bull., 17, 994-999.

JOSUKA K., ADACHI H., 1979. Environmental physiology on the $\mathrm{pH}$ tolerance of teleost. 1- Some inorganic factors affecting the survival of medaka, Oryzias latipes, exposed to low $\mathrm{pH}$ environment. Jap. J. Ecol., 29, 221-227.

KOMM S. G., REZNICHENKO P. N., 1963. L'activité motrice embryonnaire des poissons osseux. Sect. Cinématrogr. expér. med. Acad. Sci. méd. U.R.S.S., Moscou n A219, Baliskaya Ylitsa.

KUDO S., 1980. Sperm penetration and the formation of a fertilization cone in the common carp egg. Develop. Growth Differ., 22, 403-414.

LOEFLER C. A., 1971. Water exchange in the pike egg. J. exp. Biol., 55, 797-811.

PETERSON R. H., DAYE P. G., METCALFE J. L., 1980. Inhibition of atlantic salmon (Salmo salar) hatching at low pH. Can. J. Fish. aquat. Sci., 37, 770-774.

PETERSON R. H., MARTIN-ROBICHAUD D. J., 1982. Water uptake by atlantic salmon ova affected by low pH. Trans am. Fish. Soc., 111, 772-774.

PLOUIDY M. G., 1982. Composition chimique des liquides d'accompagnement des gamètes de carpe. Diplôme Fin d'Etudes de l'Institut Agricole de Beauvais.

POTTS W. T. W., RUDY P., 1969. Water balance in the egg of the atlantic salmo Salmon salar. J. exp. Biol., 50, 223-238.

RASK M., 1983. The effect of low pH on perch Perca fluviatilis L. I- Effects of low pH on the development of eggs of perch. Ann. zool. fennici, 20, 73-76.

RIDGWAY E. B., GILKEY J. C., JAFFE L. F., 1977. Free calcium increases explosively in activating medaka eggs. Proc. nat. Acad. Sci. U.S.A., 74, 623-627.

ROMBOUGH P. J., 1983. Effects of low pH on eyed embryos and alevins of pacific salmon. Can. J. Fish. aquat. Sci, 40, 1575-1582.

Roubaud P., GILLet Ch., BILLARD R., 1984. Influence du $\mathrm{pH}$ du milieu au cours de la fécondation sur la survie embryonnaire de la carpe commune (Cyprinus carpio). J. can. Zool., 62, 851-861.

ROUBAUD P., CHAILLOU C., SJAFEI D., 1985. Variations cycliques de la tolérance à un choc thermique froid appliqué au cours de la segmentation de l'embryon de la carpe commune (Cyprinus carpio L.). Can. J. Zool., 63, 657-663.

ROTT N. M., 1979. Cell cycle in early embryogenesis of whitefish. Ontogenez, 11, 3-23.

ROTT N. M., 1970. Cell division during pregastrulation period of development. Ontogenez, 11, 323.

SNSF PROJECT, 1980. Proc. int. Conf. the ecological impact of acid precipitation. Sandefjord Norway. D. Drablos et A. Tollan eds., Oslo.

SOIN S., 1977. Some features of the development of the carp (Cyprinus carpio) under hatchery conditions. J. Ichthyol., 5, 759-769.

THOMOPOULOS A., 1953. Sur I'œuf de Perca fluviatilis L. Bull Soc. Zool., Fr., 78, 106-114.

VON RAUEN H. M., 1964. Biochemisches Taschenbuch. Springer-Verlag. Berlin, pp. 37-110. 